\title{
Pion-assisted charmed dibaryon candidate
}

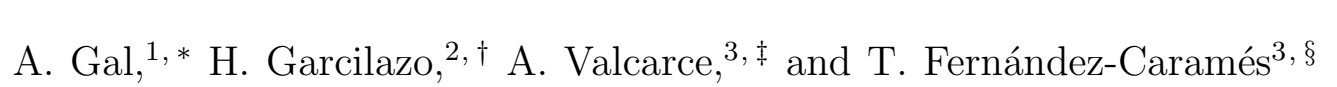 \\ ${ }^{1}$ Racah Institute of Physics, The Hebrew University, Jerusalem 91904, Israel \\ ${ }^{2}$ Escuela Superior de Física y Matemáticas \\ Instituto Politécnico Nacional, Edificio 9, 07738 México D.F., Mexico \\ ${ }^{3}$ Departamento de Física Fundamental, \\ Universidad de Salamanca, E-37008 Salamanca, Spain
}

(Dated: October 24, 2018)

\begin{abstract}
The $\Lambda_{c}(2286) N$ system is studied in a chiral constituent quark model and the resulting $s$-wave interaction is used in separable form within three-body models of the $\pi \Lambda_{c} N$ system with quantum numbers $\left(C, I, J^{P}\right)=\left(+1, \frac{3}{2}, 2^{+}\right)$. Separable interactions are also used for the dominant $p$-wave pion-baryon channels dominated by the $\Delta(1232)$ and $\Sigma_{c}(2520)$ resonances. Faddeev equations with relativistic kinematics are solved on the real axis to search for bound states and in the complex plane to search for three-body resonances. Some of the models considered generate a very narrow bound state, requiring isospin violation for its strong decay. Other models lead to a narrow resonance $(\Gamma \lesssim 0.4 \mathrm{MeV})$ for resonance mass below the $\Sigma_{c}(2455) N$ threshold. This would be the lowest-lying $C=+1$ dibaryon, with mass estimated as $\approx 3370 \pm 15 \mathrm{MeV}$.
\end{abstract}

PACS numbers: 12.39.Pn, 13.75.Gx, 14.20.Lq, 11.80.Jy

Keywords: Quark models, pion-baryon interactions, charmed baryons, Faddeev equations

*Electronic address: avragal@savion.huji.ac.il

†Electronic address: humberto@esfm.ipn.mx

${ }^{\ddagger}$ Electronic address: valcarce@fis.usal.es

$\S$ Electronic address: carames@usal.es 


\section{INTRODUCTION}

Several pion-assisted dibaryon candidates of the type $\pi B B^{\prime}$, with a $p$-wave pion interacting with baryons $B$ and $B^{\prime}$ that interact in $s$ waves, were suggested in Ref. [1]. Considered in detail was the $\pi \Lambda N$ system in the channel $\left(I, J^{P}\right)=\left(\frac{3}{2}, 2^{+}\right)$which is dominated by configurations where the $p$-wave $\pi N\left(I, J^{P}\right)=\left(\frac{3}{2}, \frac{3}{2}^{+}\right) \Delta(1232)$ resonance is coupled to an $s$-wave $\Lambda$, and the $p$-wave $\pi \Lambda\left(I, J^{P}\right)=\left(1, \frac{3}{2}^{+}\right) \Sigma(1385)$ resonance is coupled to an $s$-wave nucleon. The choice $\left(I, J^{P}\right)=\left(\frac{3}{2}, 2^{+}\right)$ensures that the spins and isospins of the three hadrons are parallel, with the two baryons necessarily in a ${ }^{3} S_{1}$ state, leading to maximal attraction since all spin and isospin recoupling coefficients in this channel are equal to one. The $\left(I, J^{P}\right)=\left(\frac{3}{2}, 2^{+}\right) \pi \Lambda N-\pi \Sigma N$ coupled-channel system was studied subsequently [2, 3] , concluding that it resonates some 10-20 MeV below the $\pi \Sigma N$ threshold [3]. Other pion-assisted dibaryon candidates suggested in [1] include $\pi \Xi N, \pi \Xi \Lambda$ and $\pi \Lambda_{c} N$. In the present work we apply the same formalism [3] to study the charmed $\pi \Lambda_{c} N$ system where one replaces the $\frac{1}{2}_{\text {g.s. }}^{+} \Lambda(1116)$ baryon by the $\frac{1}{2}_{\text {g.s. }}^{+} \Lambda_{c}(2286)$ charmed baryon, and the $\frac{3}{2}^{+} \Sigma(1385)$ resonance by the $\frac{3}{2}^{+} \Sigma_{c}(2520)$ charmed resonance. An interesting aspect of this $\pi \Lambda_{c} N$ system is that a bound state, if occurring, will decay only by isospin-violating interactions since the lowest isospin-conserving decay channel $\Sigma_{c}(2455) N$ lies $\approx 30 \mathrm{MeV}$ above the $\pi \Lambda_{c} N$ threshold.

To formulate and solve a $\pi \Lambda_{c} N$ three-body model one needs to specify the input pairwise interactions. Whereas the construction of $p$-wave separable interactions describing the pion-baryon $\Delta(1232)$ and $\Sigma_{c}(2520)$ resonances is straightforward, the construction of the necessary $s$-wave separable interaction describing the $\Lambda_{c}(2286) N$ system requires special attention. In the present exploratory study we neglect its coupling to the $\Sigma_{c}(2455) N$ system, reporting briefly on a straightforward application of the chiral constituent quark model (CCQM) within the charm sector [4, 5]. This model, tuned by fitting to the baryon and meson spectra as well as to the $N N$ interaction, provides predictions for charm $C=+1$ two-hadron systems that will become testable in due course. For an extensive review of the CCQM, see Ref. [6].

The paper is organized as follows. The input pion-baryon phenomenological interactions are discussed in Sect. [II, and the input $\Lambda_{c} N$ CCQM interactions are discussed in Sect. [II], Results of three-body calculations using Faddeev equations with relativistic kinematics are given and discussed in Sect. IV, with conclusions drawn in the last Sect. V. 


\section{PION-BARYON $p$-WAVE INTERACTIONS}

Following the discussion of the $\pi \Lambda N$ system in Ref. [1], the dominant two-body interactions in the $\pi \Lambda_{c} N$ system are the $p$-wave $\pi N\left(I, J^{P}\right)=\left(\frac{3}{2}, \frac{3}{2}^{+}\right) \Delta(1232)$ and $\pi \Lambda_{c}$ $\left(I, J^{P}\right)=\left(1, \frac{3}{2}^{+}\right) \Sigma_{c}(2520)$ channels, and the $s$-wave $\Lambda_{c} N$ interaction in the $I=\frac{1}{2},{ }^{3} S_{1}$ channel. In this section we describe the appropriate separable-interaction meson-baryon models, assigning particle indices 1,2,3 to charmed-hyperons, nucleon and pion, respectively.

\section{A. The $\pi N$ subsystem}

The Lippmann-Schwinger equation for the pion-nucleon interaction is given by [7]:

$$
\begin{aligned}
t_{1}\left(p_{1}, p_{1}^{\prime} ; \omega_{0}\right) & =V_{1}\left(p_{1}, p_{1}^{\prime}\right)+\int_{0}^{\infty} p_{1}^{\prime 2} d p_{1}^{\prime \prime} \\
& \times V_{1}\left(p_{1}, p_{1}^{\prime \prime}\right) \frac{1}{\omega_{0}-\sqrt{m_{N}^{2}+p_{1}^{\prime \prime 2}}-\sqrt{m_{\pi}^{2}+p_{1}^{\prime \prime 2}}+\mathrm{i} \epsilon} t_{1}\left(p_{1}^{\prime \prime}, p_{1}^{\prime} ; \omega_{0}\right),
\end{aligned}
$$

so that using a separable potential

$$
V_{1}\left(p_{1}, p_{1}^{\prime}\right)=\gamma_{1} g_{1}\left(p_{1}\right) g_{1}\left(p_{1}^{\prime}\right)
$$

one gets

$$
t_{1}\left(p_{1}, p_{1}^{\prime} ; \omega_{0}\right)=g_{1}\left(p_{1}\right) \tau_{1}\left(\omega_{0}\right) g_{1}\left(p_{1}^{\prime}\right)
$$

where

$$
\left[\tau_{1}\left(\omega_{0}\right)\right]^{-1}=\frac{1}{\gamma_{1}}-\int_{0}^{\infty} p_{1}^{2} d p_{1} \frac{g_{1}^{2}\left(p_{1}\right)}{\omega_{0}-\sqrt{m_{N}^{2}+p_{1}^{2}}-\sqrt{m_{\pi}^{2}+p_{1}^{2}}+\mathrm{i} \epsilon} .
$$

A fit to the $P_{33}$ phase shift and scattering volume using the form factor

$$
g_{1}\left(p_{1}\right)=p_{1}\left[\exp \left(-p_{1}^{2} / \beta_{1}^{2}\right)+C p_{1}^{2} \exp \left(-p_{1}^{2} / \alpha_{1}^{2}\right)\right]
$$

with parameters given in Table I, was shown and discussed in Ref. [7]. Listed in the table are also the r.m.s. radii of the form factors $g(p)$ in momentum space and $\tilde{g}(r)$ in coordinate space, where $\tilde{g}(\vec{r})=\hat{r} \tilde{g}(r)$ is the Fourier transform of the $p$-wave form factor $g(\vec{p})=\hat{p} g(p)$, given by

$$
\tilde{g}(r) \sim \int j_{1}(p r) g(p) p^{2} d p
$$

with $j_{1}$ the spherical Bessel function for $\ell=1$. As elaborated in Ref. [7], $\tilde{g}(r)$ is not positive definite, which may result in negative values of $<r^{2}>$. A spatial-size substitute for $\sqrt{<r^{2}>}$ 
is provided then by $r_{0}^{(\pi N)}$, the first zero of $\tilde{g}(r)$. Both values of $\sqrt{<r^{2}>}$ and $r_{0}$ listed in Table $\amalg$ are seen to be close to each other, but this need not necessarily be the case for other subsystems, as will become evident in the next subsection.

TABLE I: Fitted parameters of the $\pi N$ separable $p$-wave interaction (2) with form factor $g_{1}(p)$ defined by Eq. (5). Listed also are values of its r.m.s. momentum $\sqrt{\left\langle p^{2}\right\rangle_{g_{1}}}\left(\mathrm{in} \mathrm{fm}^{-1}\right)$, and r.m.s. radius $\sqrt{\left.<r^{2}\right\rangle_{g_{1}}}$ and zero $r_{0}^{(\pi N)}$ (both in fm) of the coordinate-space form factor $\tilde{g}_{1}(r)$.

\begin{tabular}{ccccccc}
\hline \hline$\gamma_{1}\left(\mathrm{fm}^{4}\right)$ & $\alpha_{1}\left(\mathrm{fm}^{-1}\right)$ & $\beta_{1}\left(\mathrm{fm}^{-1}\right)$ & $C\left(\mathrm{fm}^{2}\right)$ & $\sqrt{<p^{2}>_{g_{1}}}$ & $\sqrt{<r^{2} \gamma_{\tilde{g}_{1}}}$ & $r_{0}^{(\pi N)}$ \\
\hline-0.075869 & 2.3668 & 1.04 & 0.23 & 4.07 & 1.47 & 1.36 \\
\hline \hline
\end{tabular}

The pion-nucleon amplitude in the three-body system with a $\Lambda_{c}$ as spectator is given by

$$
t_{1}\left(p_{1}, p_{1}^{\prime} ; W_{0}, q_{1}\right)=g_{1}\left(p_{1}\right) \tau_{1}\left(W_{0}, q_{1}\right) g_{1}\left(p_{1}^{\prime}\right)
$$

where $W_{0}$ is the invariant mass of the three-body system, $q_{1}$ is the relative momentum between the spectator and the c.m. of the $\pi N$ subsystem and

$$
\left[\tau_{1}\left(W_{0}, q_{1}\right)\right]^{-1}=\frac{1}{\gamma_{1}}-\int_{0}^{\infty} p_{1}^{2} d p_{1} \frac{g_{1}^{2}\left(p_{1}\right)}{W_{0}-\sqrt{\left(\sqrt{m_{N}^{2}+p_{1}^{2}}+\sqrt{m_{\pi}^{2}+p_{1}^{2}}\right)^{2}+q_{1}^{2}}-\sqrt{m_{\Lambda_{c}}^{2}+q_{1}^{2}}+\mathrm{i} \epsilon} .
$$

\section{B. The $\pi \Lambda_{c}$ subsystem}

Here, the separable potential

$$
V_{2}\left(p_{2}, p_{2}^{\prime}\right)=\gamma_{2} g_{2}\left(p_{2}\right) g_{2}\left(p_{2}^{\prime}\right)
$$

is used with the form factor

$$
g_{2}\left(p_{2}\right)=p_{2}\left(1+A p_{2}^{2}\right) \exp \left(-p_{2}^{2} / \beta_{2}^{2}\right)
$$

where the three parameters $\gamma_{2}, \beta_{2}$ and $A$ were fitted to the two pieces of data available, namely, the position and width of the $\Sigma_{c}(2520)$ resonance [8]. A family of such fitted parameters is given in Table II. Scanning over $A$ between 0 and 1 gave unrealistically small positive values of $\left\langle r^{2}>_{\tilde{g_{2}}}\right.$ associated with the form factor $g_{2}$, decreasing rapidly with $A$ and becoming negative for $A$ exceeding 0.2 . Our alternative choice of $r_{0}$ for a size parameter 
gives values monotonically increasing from 1 to 1.5 upon increasing $A \neq 0$. Anticipating $r_{0}^{\left(\pi \Lambda_{c}\right)}$ to somewhat exceed $r_{0}^{(\pi N)}=1.36 \mathrm{fm}$ (Table II), because the pionic $\Lambda_{c} \rightarrow \Sigma_{c} p$-wave excitation energy of $231.5 \mathrm{MeV}$ is smaller than the corresponding excitation energy $293 \mathrm{MeV}$ for $N \rightarrow \Delta$, we consider the last two rows in Table II as the most physically acceptable fits. For further discussion of form-factor sizes, see Ref. [7].

TABLE II: Fitted parameters of the $\pi \Lambda_{c}$ separable $p$-wave interaction defined by Eqs. (9) and (10), for chosen values of the parameter $A$. Listed also are values of the r.m.s. momentum $\sqrt{\left\langle p^{2}\right\rangle_{g_{2}}}$ (in $\mathrm{fm}^{-1}$ ), the r.m.s. radius $\sqrt{<r^{2}>\tilde{g}_{2}}$ and zero $r_{0}^{\left(\pi \Lambda_{c}\right)}$ (both in fm) of the Fourier transform $\tilde{g_{2}}(r)$.

\begin{tabular}{|c|c|c|c|c|c|}
\hline$A\left(\mathrm{fm}^{2}\right)$ & $\gamma_{2}\left(\mathrm{fm}^{4}\right)$ & $\beta_{2}\left(\mathrm{fm}^{-1}\right)$ & $\sqrt{<p^{2}>_{g_{2}}}$ & $\sqrt{<r^{2}>\tilde{g_{2}}}$ & $r_{0}^{\left(\pi \Lambda_{c}\right)}$ \\
\hline 0.0 & -0.0044983 & 6.4738 & 9.155 & 0.437 & - \\
\hline 0.1 & -0.0057655 & 3.6951 & 6.108 & 0.332 & 0.973 \\
\hline 0.2 & -0.0062314 & 3.1432 & 5.258 & 0.070 & 1.103 \\
\hline 0.3 & -0.0063429 & 2.8568 & 4.806 & - & 1.194 \\
\hline 0.4 & -0.0062715 & 2.6737 & 4.515 & - & 1.263 \\
\hline 0.5 & -0.0061012 & 2.5441 & 4.307 & - & 1.318 \\
\hline 0.7 & -0.0056362 & 2.3695 & 4.026 & - & 1.401 \\
\hline 1.0 & -0.0048792 & 2.2121 & 3.772 & - & 1.487 \\
\hline
\end{tabular}

\section{THE $\Lambda_{c} N$ SUBSYSTEM}

There is no experimental data on the $\Lambda_{c} N$ subsystem that one may rely upon to fit a separable potential form. Therefore, and as a guide, we have generated local potentials in the $I=\frac{1}{2},{ }^{3} S_{1}$ channel from the recent application of the CCQM to the charmed meson sector [5]. A brief description of the essential properties required in this model to provide interaction output for the $\Lambda_{c} N$ system follows.

\section{A. Extension of the CCQM to the charm sector}

Baryons are described in the CCQM as clusters of three interacting massive constituent quarks, with the light-quark $(u, d)$ mass generated by the spontaneously broken 
$S U(2)_{L} \otimes S U(2)_{R}$ chiral symmetry of the QCD Lagrangian. Hence, light quarks interact nonperturbatively via Nambu-Goldstone boson-exchange potentials

$$
V_{\chi}\left(\vec{r}_{i j}\right)=V_{\mathrm{OSE}}\left(\vec{r}_{i j}\right)+V_{\mathrm{OPE}}\left(\vec{r}_{i j}\right)
$$

given in obvious notation by

$$
\begin{gathered}
V_{\mathrm{OSE}}\left(\vec{r}_{i j}\right)=-\frac{g_{\mathrm{ch}}^{2}}{4 \pi} \frac{\Lambda^{2}}{\Lambda^{2}-m_{\sigma}^{2}} m_{\sigma}\left[Y\left(m_{\sigma} r_{i j}\right)-\frac{\Lambda}{m_{\sigma}} Y\left(\Lambda r_{i j}\right)\right] \\
V_{\mathrm{OPE}}\left(\vec{r}_{i j}\right)=\frac{g_{\mathrm{ch}}^{2}}{4 \pi} \frac{m_{\pi}^{2}}{12 m_{i} m_{j}} \frac{\Lambda^{2}}{\Lambda^{2}-m_{\pi}^{2}} m_{\pi}\left\{\left[Y\left(m_{\pi} r_{i j}\right)-\frac{\Lambda^{3}}{m_{\pi}^{3}} Y\left(\Lambda r_{i j}\right)\right] \vec{\sigma}_{i} \cdot \vec{\sigma}_{j}\right. \\
\left.+\left[H\left(m_{\pi} r_{i j}\right)-\frac{\Lambda^{3}}{m_{\pi}^{3}} H\left(\Lambda r_{i j}\right)\right] S_{i j}\right\} \vec{\tau}_{i} \cdot \vec{\tau}_{j},
\end{gathered}
$$

where $g_{\mathrm{ch}}^{2} / 4 \pi$ is the chiral coupling constant, $Y(x)$ is the Yukawa function, $Y(x)=e^{-x} / x$, and $H(x)=\left(1+3 / x+3 / x^{2}\right) Y(x)$ is associated with the quark-quark tensor operator $S_{i j}=3\left(\vec{\sigma}_{i} \cdot \hat{r}_{i j}\right)\left(\vec{\sigma}_{j} \cdot \hat{r}_{i j}\right)-\vec{\sigma}_{i} \cdot \vec{\sigma}_{j}$. The values used for the mass, coupling-constant and cut-off parameters are listed in Table 2 of [5]. In the case of the heavy charmed quark $c$, for which chiral symmetry is explicitly broken, no boson-exchange is operative in its interactions with the other quarks.

Perturbative effects within QCD are accounted for by the one-gluon-exchange (OGE) potential

$$
V_{\mathrm{OGE}}\left(\vec{r}_{i j}\right)=\frac{\alpha_{s}}{4} \vec{\lambda}_{i}^{\mathrm{c}} \cdot \vec{\lambda}_{j}^{\mathrm{c}}\left[\frac{1}{r_{i j}}-\frac{1}{4}\left(\frac{1}{2 m_{i}^{2}}+\frac{1}{2 m_{j}^{2}}+\frac{2 \vec{\sigma}_{i} \cdot \vec{\sigma}_{j}}{3 m_{i} m_{j}}\right) \frac{e^{-r_{i j} / r_{0}}}{r_{0}^{2} r_{i j}}-\frac{3 S_{i j}}{4 m_{q}^{2} r_{i j}^{3}}\right],
$$

where $\lambda^{c}$ are the $S U(3)$ color matrices, $r_{0}$ is a flavor-dependent regularization that scales with the reduced mass of the interacting pair, and $\alpha_{s}$ is the QCD scale-dependent coupling constant which assumes values of $\alpha_{s} \sim 0.54$ for light-quark pairs and $\alpha_{s} \sim 0.43$ for $u c$ and dc pairs [4].

Finally, to fully simulate QCD one needs to incorporate confinement. While negligible for hadron-hadron interactions, lattice calculations suggest that the confinement potential is screened upon increasing the interquark distance [9],

$$
V_{\mathrm{CON}}\left(\vec{r}_{i j}\right)=\left[-a_{c}\left(1-e^{-\mu_{c} r_{i j}}\right)\right] \overrightarrow{\lambda c}_{i} \cdot \overrightarrow{\lambda c}_{j}
$$

with a scale given by $a_{c}=230 \mathrm{MeV}$ and a screening mass identified here with the pion mass: $\mu_{c}=m_{\pi}$. 
The CCQM yields a good description of meson [4] and baryon spectra [10]. Furthermore, by applying resonating-group methods it enables one to derive baryon-baryon $(B B)$ potentials and, in particular, to reproduce the main features of the $N N$ interaction [6]. Thus, the $B_{n} B_{m} \rightarrow B_{k} B_{l}$ local transition potential $V_{B_{n} B_{m}(L S T) \rightarrow B_{k} B_{l}\left(L^{\prime} S^{\prime} T\right)}(R)$ is derived within a Born-Oppenheimer approximation as

$$
V_{B_{n} B_{m}(L S T) \rightarrow B_{k} B_{l}\left(L^{\prime} S^{\prime} T\right)}(R)=\xi_{L S T}^{L^{\prime} S^{\prime} T}(R)-\xi_{L S T}^{L^{\prime} S^{\prime} T}(\infty),
$$

where

$$
\xi_{L S T}^{L^{\prime} S^{\prime} T}(R)=\frac{\left\langle\Psi_{B_{k} B_{l}}^{L^{\prime} S^{\prime} T}(\vec{R})\left|\sum_{i<j=1}^{6} V_{q_{i} q_{j}}\left(\vec{r}_{i j}\right)\right| \Psi_{B_{n} B_{m}}^{L S T}(\vec{R})\right\rangle}{\sqrt{\left\langle\Psi_{B_{k} B_{l}}^{L^{\prime} S^{\prime} T}(\vec{R}) \mid \Psi_{B_{k} B_{l}}^{L^{\prime} S^{\prime} T}(\vec{R})\right\rangle} \sqrt{\left\langle\Psi_{B_{n} B_{m}}^{L S T}(\vec{R}) \mid \Psi_{B_{n} B_{m}}^{L S T}(\vec{R})\right\rangle}}
$$

with the quark coordinates integrated out. The wavefunction $\Psi_{B_{n} B_{m}}^{L S T}(\vec{R})$ for the two-baryon system is an antisymmetrized product of two three-quark clusters, each cluster consisting of Gaussian quark wavefunctions. The Gaussian size parameters from Table 2 in Ref. [5] are $b_{n}=0.518 \mathrm{fm}$ for the $(u, d)$ light quarks, here denoted $n$, and $b_{c}=0.6 \mathrm{fm}$ for the $c$ quark. However, whereas the value adopted for $b_{n}$ was deduced long ago by fitting to the $N N$ phase shifts and the deuteron binding energy [11] (see also the discussion in Ref. [12]), the value $b_{c}=0.6 \mathrm{fm}$ is not constrained by any comparable $B B$ data. It was argued in Ref. [13] that a considerably smaller value of $b_{c}$, in fact $b_{c} \approx 0.2 \mathrm{fm}$, is required to describe consistently doubly charmed exotic mesons. Such a value may also be justified by recalling that $b_{q}$ scales with quark-mass as $b_{q} \sim m_{q}^{-1 / 2}$ for harmonic-oscillator quark potential. For CCQM quark masses $m_{n}=313 \mathrm{MeV}$ and $m_{c}=1752 \mathrm{MeV}$, the widely adopted value $b_{n}=0.518 \mathrm{fm}$ implies that $b_{c}=b_{n}\left(m_{n} / m_{c}\right)^{1 / 2}=0.219 \mathrm{fm}$. This strong dependence on the constituent quark mass by far overshadows the weak flavor dependence of the harmonic-oscillator $1 \hbar \omega$ excitation energy, of order hundreds of $\mathrm{MeV}$, in mesons and in baryons.

\section{B. The CCQM $I=\frac{1}{2},{ }^{3} S_{1} \Lambda_{c} N$ interaction}

Adopting the CCQM $s$-wave potentials for the $\Lambda_{c} N$ interacting pair, we show on the l.h.s. of Fig. 1 three such potentials for the $I=\frac{1}{2},{ }^{3} S_{1}$ channel, using three different values of the charmed quark oscillator parameter $b_{c}$ within the six-quark wave function: $b_{c}=0.2 \mathrm{fm}$, $b_{c}=0.5 \mathrm{fm}$ and $b_{c}=0.8 \mathrm{fm}$. It is seen that the model with $b_{c}=0.2 \mathrm{fm}$ has the softest 

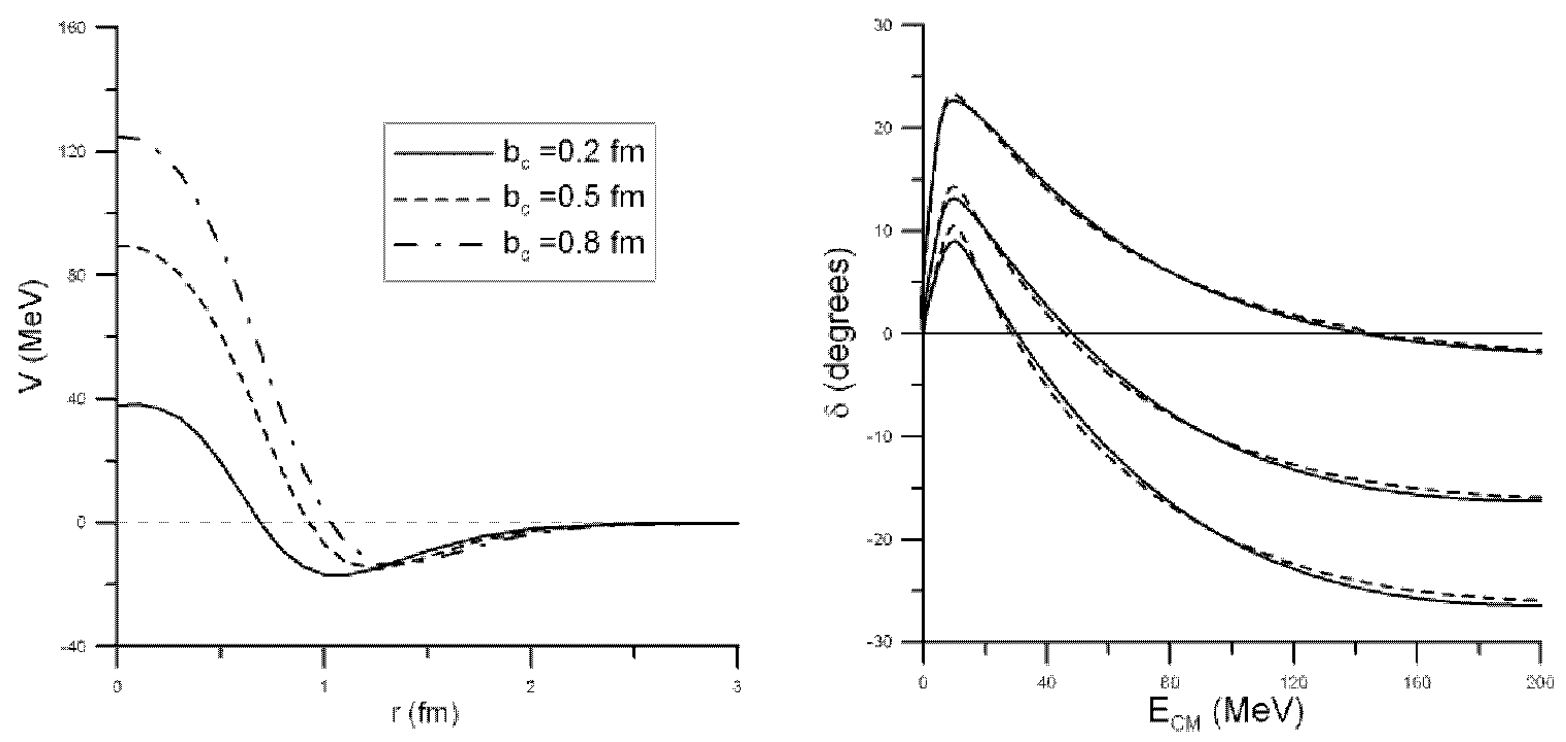

FIG. 1: Left: CCQM $\Lambda_{c} N$ local potentials in the ${ }^{3} S_{1}$ channel for several values of the charmed quark harmonic oscillator size parameter $b_{c}$. Right: corresponding $\Lambda_{c} N^{3} S_{1}$ phase shifts, in solid (dashed) lines, produced by the CCQM local (separable) potentials. The smaller $b_{c}$ is, the higher is the maximum of the phase shift $\delta$.

repulsive core and the model with $b_{c}=0.8 \mathrm{fm}$ has the strongest repulsion at short distance. None of these models is any close to generating a $\Lambda_{c} N$ bound state. The ${ }^{3} S_{1}$ phase shifts produced by these potentials are shown by the solid lines on the r.h.s. of the figure where the change of sign of the phase shift for the model with $b_{c}=0.2 \mathrm{fm}$ occurs at c.m. energy similar to that of other baryon-baryon systems in the CCQM such as $N N$ or $\Lambda N$, while for the models with $b_{c}=0.5$ and $b_{c}=0.8 \mathrm{fm}$ the change of sign occurs at very low c.m. energy which suggests that these latter two models have excessive repulsion. We also show by the dashed lines the phase shifts obtained using the rank- 2 separable potential with both attraction and repulsion

$$
V_{3}\left(p_{3}, p_{3}^{\prime}\right)=-g_{3}^{a}\left(p_{3}\right) g_{3}^{a}\left(p_{3}^{\prime}\right)+g_{3}^{r}\left(p_{3}\right) g_{3}^{r}\left(p_{3}^{\prime}\right)
$$

so that the corresponding two-body $t$-matrix is given by

$$
t_{3}\left(p_{3}, p_{3}^{\prime} ; \omega_{0}\right)=-\sum_{\alpha=a, r} \sum_{\beta=a, r} g_{3}^{\alpha}\left(p_{3}\right) \tau_{3}^{\alpha \beta}\left(\omega_{0}\right) g_{3}^{\beta}\left(p_{3}^{\prime}\right)
$$


where

$$
\begin{gathered}
\tau_{3}^{a r}\left(\omega_{0}\right)=\tau_{3}^{r a}\left(\omega_{0}\right)=\frac{G_{3}^{a r}\left(\omega_{0}\right)}{\left[1+G_{3}^{a a}\left(\omega_{0}\right)\right]\left[1-G_{3}^{r r}\left(\omega_{0}\right)\right]+\left[G_{3}^{a r}\left(\omega_{0}\right)\right]^{2}}, \\
\tau_{3}^{a a}\left(\omega_{0}\right)=\frac{1-G_{3}^{r r}\left(\omega_{0}\right)}{\left[1+G_{3}^{a a}\left(\omega_{0}\right)\right]\left[1-G_{3}^{r r}\left(\omega_{0}\right)\right]+\left[G_{3}^{a r}\left(\omega_{0}\right)\right]^{2}}, \\
\tau_{3}^{r r}\left(\omega_{0}\right)=-\frac{1+G_{3}^{a a}\left(\omega_{0}\right)}{\left[1+G_{3}^{a a}\left(\omega_{0}\right)\right]\left[1-G_{3}^{r r}\left(\omega_{0}\right)\right]+\left[G_{3}^{a r}\left(\omega_{0}\right)\right]^{2}},
\end{gathered}
$$

with $G_{3}^{\alpha \beta}\left(\omega_{0}\right)$ given by

$$
G_{3}^{\alpha \beta}\left(\omega_{0}\right)=\int_{0}^{\infty} p_{3}^{2} d p_{3} \frac{g_{3}^{\alpha}\left(p_{3}\right) g_{3}^{\beta}\left(p_{3}\right)}{\omega_{0}-\sqrt{p_{3}^{2}+m_{N}^{2}}-\sqrt{p_{3}^{3}+m_{\Lambda_{c}}^{2}}+i \epsilon} .
$$

The form factors $g_{3}^{\beta}\left(p_{3}\right)$ are chosen to be of the Yamaguchi form

$$
g_{3}^{\beta}\left(p_{3}\right)=\frac{\sqrt{\gamma_{\beta}}}{p_{3}^{2}+\alpha_{\beta}^{2}} \quad(\beta=a, r)
$$

and the parameters of these models are given in Table III together with values of the associated scattering lengths and effective ranges. The relatively small size of the scattering lengths $a_{\Lambda_{c} N}$ clearly indicates that the ${ }^{3} S_{1} \Lambda_{c} N$ system is far from binding on its own.

TABLE III: Parameters of the ${ }^{3} S_{1} \Lambda_{c} N$ separable potential models Eqs. (18), (24).

\begin{tabular}{ccccccc}
\hline \hline$b_{c}(\mathrm{fm})$ & $\gamma_{3}^{a}\left(\mathrm{fm}^{2}\right)$ & $\alpha_{3}^{a}\left(\mathrm{fm}^{-1}\right)$ & $\gamma_{3}^{r}\left(\mathrm{fm}^{2}\right)$ & $\alpha_{3}^{r}\left(\mathrm{fm}^{-1}\right)$ & $a(\mathrm{fm})$ & $r(\mathrm{fm})$ \\
\hline 0.2 & 1.8915 & 1.7672 & 2.6210 & 2.1523 & -1.33 & 3.3 \\
0.5 & 2.1804 & 1.7030 & 4.5651 & 2.1973 & -0.79 & 5.6 \\
0.8 & 2.3435 & 1.6286 & 5.7197 & 2.1338 & -0.63 & 8.2 \\
\hline \hline
\end{tabular}

\section{RESULTS AND DISCUSSION}

Solutions of the Faddeev equations corresponding to bound states and resonance poles in the $\left(I, J^{P}\right)=\left(\frac{3}{2}, 2^{+}\right)$channel of the $\pi \Lambda_{c} N$ three-body system were found applying search procedures described in Refs. [1 3] . A single bound state or resonance was established for any combination of each one of the $\pi \Lambda_{c}$ interaction models specified in Table II and each one of the $\Lambda_{c} N$ interaction models specified in Table【II, as well as for the case when there is no $\Lambda_{c} N$ interaction. The resulting bound-state and resonance energies are given in Table IV with respect to the $\pi \Lambda_{c} N$ threshold mass $E_{\mathrm{th}} \approx 3363 \mathrm{MeV}$. 
TABLE IV: Energy eigenvalue of the $\left(I, J^{P}\right)=\left(\frac{3}{2}, 2^{+}\right) \pi \Lambda_{c} N$ state (in MeV with respect to the $\pi \Lambda_{c} N$ threshold) for the eight models of the $\pi \Lambda_{c}$ interaction characterized by the parameter $A$ and the three models of the $\Lambda_{c} N$ interaction $b_{c}=0.2, b_{c}=0.5$, and $b_{c}=0.8 \mathrm{fm}$, as well as for $V_{\Lambda_{c} N}=0$.

\begin{tabular}{ccccc}
\hline \hline$A\left(\mathrm{fm}^{2}\right)$ & $b_{c}=0.2$ & $b_{c}=0.5$ & $b_{c}=0.8$ & $V_{\Lambda_{c} N}=0$ \\
\hline 0.0 & -2.3 & $36.7-\mathrm{i} 0.45$ & $63.1-\mathrm{i} 1.91$ & -0.7 \\
0.1 & -14.6 & $26.3-\mathrm{i} 0.18$ & $54.8-\mathrm{i} 1.16$ & -12.5 \\
0.2 & -12.7 & $27.2-\mathrm{i} 0.21$ & $55.2-\mathrm{i} 1.22$ & -10.1 \\
0.3 & -8.4 & $30.0-\mathrm{i} 0.28$ & $57.0-\mathrm{i} 1.53$ & -5.3 \\
0.4 & -4.0 & $33.1-\mathrm{i} 0.38$ & $59.1-\mathrm{i} 1.67$ & -0.5 \\
0.5 & $0.1-\mathrm{i} 0.00$ & $35.9-\mathrm{i} 0.51$ & $61.2-\mathrm{i} 1.96$ & $3.9-\mathrm{i} 0.00$ \\
0.7 & $7.0-\mathrm{i} 0.01$ & $40.8-\mathrm{i} 0.80$ & $64.4-\mathrm{i} 2.34$ & $11.3-\mathrm{i} 0.01$ \\
1.0 & $14.7-\mathrm{i} 0.07$ & $46.2-\mathrm{i} 1.10$ & $68.2-\mathrm{i} 3.05$ & $19.5-\mathrm{i} 0.07$ \\
\hline \hline
\end{tabular}

Bound-state solutions appear in several of the $b_{c}=0.2 \mathrm{fm}$ models and also when the $\Lambda_{c} N$ interaction is switched off, whereas the models $b_{c}=0.5$ and $b_{c}=0.8 \mathrm{fm}$ give only resonance solutions. However, when the resonance lies below the $\Sigma_{c} N$ threshold ( $\operatorname{Re} E<27 \mathrm{MeV}$ ) the resonance states are quite narrow with widths less than $0.4 \mathrm{MeV}$. This does not apply to the models with $b_{c}=0.5$ and $b_{c}=0.8 \mathrm{fm}$ in which the resonance lies above the $\Sigma_{c}(2455) N$ threshold and its width is therefore larger than indicated by the tabulated widths.

As concluded in Sect. IIB, only values of $A>0.5 \mathrm{fm}^{2}$ are acceptable in considering the $\Lambda_{c}(2286)+\pi \rightarrow \Sigma_{c}(2520) p$-wave form factor relative to the $N(939)+\pi \rightarrow \Delta(1232) p$-wave form factor. Combined with the more plausible $\Lambda_{c}(2286) N$ interaction model defined by choosing $b_{c}=0.2 \mathrm{fm}$, or even neglecting for simplicity this $\Lambda_{c} N$ interaction, we conclude from Table IV that the $\left(I, J^{P}\right)=\left(\frac{3}{2}, 2^{+}\right)$state of the $\pi \Lambda_{c} N$ system resonates at energy up to about $20 \mathrm{MeV}$ above threshold, that is at $3363 \lesssim \sqrt{s} \lesssim 3383 \mathrm{MeV}$. This resonance is expected to be quite narrow, with width less than $0.2 \mathrm{MeV}$ dominated by its elastic width. Pion absorption can occur only by violating charge independence, $\left(I_{\pi \Lambda_{c} N}=\frac{3}{2}\right) \rightarrow\left(I_{\Lambda_{c} N}=\frac{1}{2}\right)$, and the lowest $\Sigma_{c} N$ channel is closed at the energy range expected for the resonance. 


\section{CONCLUSION}

It was demonstrated in this work by solving Faddeev equations that the $\left(I, J^{P}\right)=\left(\frac{3}{2}, 2^{+}\right)$ state of the $\pi \Lambda_{c} N$ system is a strong candidate for a pion-assisted charmed dibaryon. Although the CCQM $\Lambda_{c} N$ interaction is not sufficiently strong to bind a $\Lambda_{c} N{ }^{3} S_{1}$ pair (and this holds even more so for a ${ }^{1} S_{0}$ pair in the CCQM), the $p$-wave pion attractive interactions induced by the $J^{P}=\frac{3}{2}^{+} \Delta(1232)$ and $\Sigma_{c}(2520)$ resonances manage to bind the $\pi \Lambda_{c} N$ threebody system or more likely to make it resonate. The prediction of this dibaryon candidate is robust in the sense that its existence depends little on the $\Lambda_{c} N$ spin-triplet $s$-wave interaction, even if the precise energy of the resonance is not pinned down between threshold at $\approx 3363 \mathrm{MeV}$ and several tens of $\mathrm{MeV}$ above threshold according to the variation offered in Table IV. This resonance is likely to be the lowest lying charmed dibaryon, considerably below the mass $\approx 3500 \mathrm{MeV}$ predicted recently for a $D N N$ bound state with quantum numbers $I=\frac{1}{2}, J^{P}=0^{-}$that may be viewed also as a $\Lambda_{c}(2595) N$ bound state [14]. These two charmed-dibaryon predictions, with $\left(I, J^{P}\right)=\left(\frac{3}{2}, 2^{+}\right)$(ours) versus $\left(I, J^{P}\right)=\left(\frac{1}{2}, 0^{-}\right)$[14], bear structural resemblance to the strange-dibaryon predictions of $\pi \Lambda N\left(I=\frac{3}{2}, J^{P}=2^{+}\right)$ [1-3] versus $K^{-} p p\left(I=\frac{1}{2}, J^{P}=0^{-}\right)$that may also be viewed as a $\Lambda(1405) N$ quasibound state [15]. None of these dibaryon candidates has been confirmed by experiment.

Denoting the $\left(I, J^{P}\right)=\left(\frac{3}{2}, 2^{+}\right) \pi \Lambda_{c} N$ dibaryon candidate by $\mathcal{Y}_{c}$, in analogy to the $\left(I, J^{P}\right)=\left(\frac{3}{2}, 2^{+}\right) \pi \Lambda N$ dibaryon candidate $\mathcal{Y}$ [15], the following production reactions of $\mathcal{Y}_{c}$ are feasible with proton and pion beams in the high-momentum hadron beam line extension approved at J-PARC:

$$
\begin{aligned}
& p+p \rightarrow \mathcal{Y}_{c}^{+++}+D^{-} \\
& \hookrightarrow \Sigma_{c}^{++}(2455)+p, \\
& \pi^{+}+d \rightarrow \quad \mathcal{Y}_{c}^{+++}+D^{-} \\
& \hookrightarrow \Sigma_{c}^{++}(2455)+p, \\
& \pi^{-}+d \rightarrow \quad \mathcal{Y}_{c}^{+}+D^{-} \\
& \hookrightarrow \Sigma_{c}^{+/ 0}(2455)+n / p
\end{aligned}
$$

The $\mathcal{Y}_{c}$ dibaryon resonance may be looked for both within inclusive missing-mass measurements by focusing on the outgoing $D^{-}$charmed meson, and in exclusive invariant-mass 
measurements focusing on the outgoing $\Sigma_{c}(2455) N$ decay pair provided that $\mathcal{Y}_{c}$ is located above the $\Sigma_{c}(2455) N$ threshold.

\section{Acknowledgments}

The research of A.G. is partially supported by the HadronPhysics3 networks SPHERE and LEANNIS of the European FP7 initiative, the research of H.G. is supported in part by COFAA-IPN (México), and the research of A.V. and T.F.C. is supported by the Spanish

Ministerio de Educación y Ciencia and EU FEDER under Contract No. FPA2010-21750, and by the Spanish Consolider-Ingenio 2010 Program CPAN (CSD2007-00042).

[1] A. Gal and H. Garcilazo, Phys. Rev. D 78, 014013 (2008).

[2] H. Garcilazo and A. Gal, Phys. Rev. C 81, 055205 (2010).

[3] H. Garcilazo and A. Gal, Nucl. Phys. A 897, 167 (2013).

[4] J. Vijande, F. Fernández, and A. Valcarce, J. Phys. G 31, 481 (2005).

[5] T. F. Caramés and A. Valcarce, Phys. Rev. D 85, 094017 (2012).

[6] A. Valcarce, H. Garcilazo, F. Fernández, and P. González, Rep. Prog. Phys. 68, 965 (2005).

[7] A. Gal and H. Garcilazo, Nucl. Phys. A 864, 153 (2011).

[8] J. Beringer et al., Phys. Rev. D 86, 010001 (2012) http://pdg.lbl.gov.

[9] G. S. Bali, Phys. Rep. 343, 1 (2001).

[10] A. Valcarce, H. Garcilazo, and J. Vijande, Phys. Rev. C 72, 025206 (2005).

[11] A. Valcarce, A. Buchmann, F. Fernández, and A. Faessler, Phys. Rev. C 50, 2246 (1994).

[12] A. Valcarce, P. González, F. Fernández, and V. Vento, Phys. Lett. B 367, 35 (1996).

[13] T. F. Caramés, A. Valcarce, and J. Vijande, Phys. Lett. B 699, 291 (2011).

[14] M. Bayar, C. W. Xiao, T. Hyodo, A. Doté, M. Oka, and E. Oset, Phys. Rev. C 86, 044004 (2012).

[15] see A. Gal, Nucl. Phys. A 914, 270 (2013) for a recent review. 